\title{
Highest electron affinity as a predictor of cluster anion structures
}

\author{
LEEOR KRONIK ${ }^{1}$, ROLAND FROMHERZ ${ }^{2}$, EUNJUNG KO ${ }^{1}$, GERD GANTEFÖR ${ }^{2}$ \& JAMES R. CHELIKOWSKY*1 \\ ${ }^{1}$ Department of Chemical Engineering and Materials Science, and Minnesota Supercomputing Institute, University of Minnesota, Minneapolis, Minnesota 55455, USA \\ ${ }^{2}$ Department of Physics, University of Konstanz, Konstanz D-78457, Germany \\ *e-mail: jrc@msi.umn.edu
}

Small clusters have a range of unique physical and chemical phenomena that are strongly size dependent. However, analysis of these phenomena often assumes that thermodynamic equilibrium conditions prevail. We compare experimentally measured and $a b$ initio computed photoelectron spectra of bare and deuterated silicon cluster anions produced in a plasma environment. We find that the isomers detected experimentally are usually not the ground-state isomers, but metastable ones, which indicates that cluster relaxation is strongly limited kinetically by a dwell time that is much shorter than the relaxation time. We show that, under these conditions, the highest electron affinity replaces the traditional lowest total energy as the appropriate criterion for predicting isomer structures. These findings demonstrate that a stringent examination of nonequilibrium effects can be crucial for a correct analysis of cluster properties.
0 mall clusters (typically comprising up to several tens of atoms) offer a 'bridge' between molecular and solid-state physics ${ }^{1,2}$. This bridge is made possible because, with increasing cluster size, all cluster properties (for example, structural, mechanical, thermal, electronic and optical) evolve from molecular values to bulk values in non-trivial ways, often revealing new and surprising physics.

A fundamental difficulty of cluster studies is that experimentally accessible quantities are often highly sensitive to the cluster structure. However, there is no known general experimental method for determining cluster structures, and indeed such structures are known experimentally only for a handful of special cases. Detailed theoretical analyses of experimental results therefore usually begin by determining cluster structures. Subsequent agreement between theory and experiment on other cluster properties is then typically invoked as evidence in support of the computed structure.

Theoretical computations of cluster structures almost invariably assume that experimental conditions allow for thermodynamic equilibrium, so that the clusters probed are very close to their ground state. Based on this paradigm, sophisticated ground-statesearching algorithms, using various forms of molecular dynamics ${ }^{3-8}$ and genetic algorithms ${ }^{9-11}$, have emerged. Such algorithms, in one form or another, are standard tools of investigation in theoretical cluster science. Even with the most advanced search algorithms, one often finds many isomers that are very close in total energy, but differ significantly in structural and other properties. In many cases, the inter-isomer energy differences are smaller than the accuracy of common tight-binding approaches, or even density functional approaches $^{9,12}$. In cases of doubt, a positive identification of the 'true' ground state requires tools that are in principle more accurate, but computationally very demanding, for example, quantum Monte Carlo (QMC) calculations ${ }^{13}$.

The fundamental assumption of thermodynamic equilibrium, which is at the heart of the above approaches, is usually taken for granted. However, its verification is by no means obvious, and little has been done to explore substitute theoretical algorithms for isomer searches and identifications in the absence of equilibrium. In this article, we pursue these questions by investigating negatively charged bare and deuterated silicon clusters, produced in a plasma environment-an important system for both basic research and 


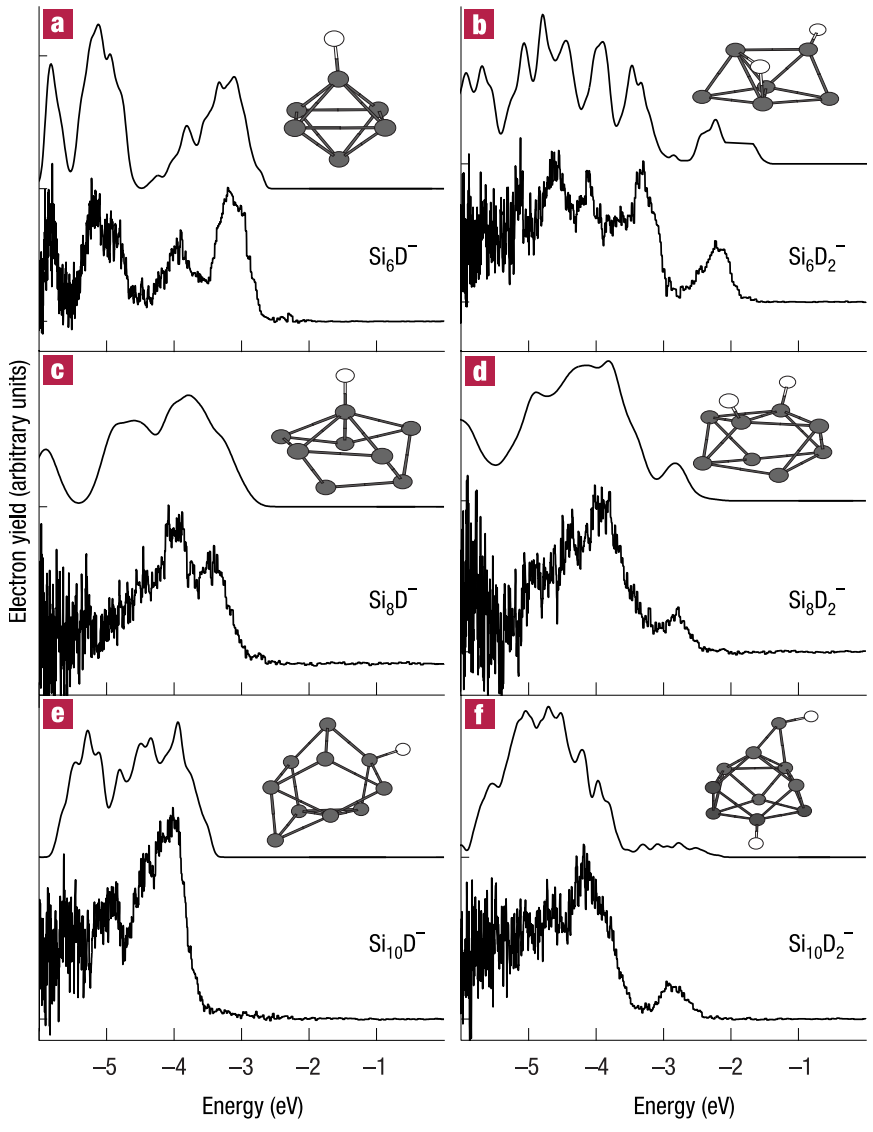

Figure 1 Photoelectron spectra (PES) of selected $\mathrm{Si}_{m} \mathrm{D}_{n}^{-}$clusters $(m=6,8$ and $10 ; n=1$ and 2). For each cluster, experimental PES data (bottom curves) are compared with theoretical PES data (top curves) taken from the isomers yielding best agreement with experiment. Isomer structures are given as insets.

technological applications. By combining photoelectron spectroscopy (PES) experiments and ab initio density functional theory (DFT) calculations, we show that cluster anion formation in this system is kinetically, rather than thermodynamically, limited. We establish that the observed clusters are metastable isomers, rather than the ground-state ones. We further show that the kinetic limitation results in a 'highest electron affinity' rule replacing the 'lowest total energy' rule as the guideline to isomer selection.

We have compared experimental and theoretical PES curves for negatively charged $\mathrm{Si}_{m} \mathrm{D}_{n}$ clusters $(4 \leq m \leq 10,0 \leq n \leq 2$, D denoting deuterium), produced using a pulsed arc cluster ion source. A selection of PES curves is given in Fig. 1. For brevity, Fig. 1 focuses on deuterated clusters $(n>0)$, and shows only the cases of $m=6,8$ and 10. In this figure, each measured spectrum is compared with the theoretical PES curve found to be in best agreement with experiment. For each cluster type, the structure of the isomer yielding the theoretical data is given as an inset. Below we refer to these isomers as "experimentally observed". The agreement between theory and experiment, in terms of both peak positions and peak intensities, is consistently good. The same level of agreement was found for all cluster types not shown in Fig. 1. This strongly suggests that our identification of the observed structures is valid. Furthermore, for the bare Si anion clusters, which have been studied extensively in the past, the various low-energy isomers we found agree with those identified in previous reports ${ }^{14-19}$. Owing to different physical approximations and different computational details (for example. choice of basis), the above reports do not always agree with each other on the relative energy ordering of these isomers. However, we find that our energy ordering for the bare Si clusters is in consistent agreement with the independent, plane-wave-based local density approximation (LDA) calculations of Wei et al. ${ }^{17}$. Information on hydrogenated Si cluster anions is much more limited, but we do find that our $\mathrm{Si}_{4} \mathrm{D}^{-}$structure agrees with the $\mathrm{Si}_{4} \mathrm{H}^{-}$suggested by $\mathrm{Xu}$ et $a l^{20}$.

For the $\mathrm{Si}_{m} \mathrm{D}_{2}{ }^{-}$clusters shown in Fig. 1, a highest occupied molecular orbital (HOMO)-lowest unoccupied molecular orbital (LUMO) gap is clearly observed, for both theory and experiment, as the separation between the independent peak at the high energy end of the spectrum and the next feature in the spectrum ${ }^{21}$. This is because these charged clusters have an odd number of electrons, so that the orbital corresponding to the LUMO in the neutral cluster is half-filled and is probed by photoemission. For the $\mathrm{Si}_{n} \mathrm{D}^{-}$clusters, the number of electrons is even and the orbital corresponding to the LUMO in the neutral cluster is empty. Consequently, the HOMOLUMO gap cannot be observed.

Figure 2 compares, for several cluster types, the PES data of the experimentally observed isomer and those for the isomer of lowest energy for which the computed PES curve disagrees with the experimental one (referred to as the "experimentally not observed" isomer below). Figure 2 also compares the structure, total energy $\left(E_{\text {tot }}\right)$ and vertical electron affinity (VEA, defined and discussed below) of those isomers. The experimental observation of a given isomer does not seem to have any correlation with minimum energy considerations. In some cases, the isomer observed is indeed the one with the lowest energy found. For example, the observed $\mathrm{Si}_{6}{ }^{-}$ isomer is lower in total energy than the first unobserved one by $\sim 0.2 \mathrm{eV}$ (Fig. 2a). In many cases, however, the observed isomer is higher in total energy than some unobserved ones, for example, by $\sim 0.3 \mathrm{eV}$ for $\mathrm{Si}_{8}{ }^{-}$(Fig. 2b) and $\sim 0.7 \mathrm{eV}$ for $\mathrm{Si}_{10} \mathrm{D}^{-}$(Fig. 2c).

We note that, contrary to both this work and ref. 17, other researchers ${ }^{16}$ designated the top $\mathrm{Si}_{6}{ }^{-}$structure in Fig. $2 \mathrm{a}$ and the bottom $\mathrm{Si}_{8}{ }^{-}$structure in Fig. $2 \mathrm{~b}$ as the ground-state ones. Even if this finding is correct, it would only serve to underscore our conclusion-the observed isomers would still not correlate with the minimum energy ones. The only difference would be that $\mathrm{Si}_{6}{ }^{-}$, rather than $\mathrm{Si}_{8}{ }^{-}$, would be the one disagreeing with the minimum energy rule.

It is unlikely that the lack of correlation between the observed isomer and the computed lowest energy one exposes a systematic failure of the LDA for several reasons. (1) For some clusters (for example, $\mathrm{Si}_{10} \mathrm{D}^{-}$) the energy differences are in the range $0.5 \mathrm{eV}$ to $1 \mathrm{eV}-\mathrm{a}$ difference much larger than the typical error made by an LDA calculation, which is about $0.1 \mathrm{eV}$. (2) Detailed QMC studies of small Si clusters have shown that, for neutral clusters, LDA gives the correct geometry and a correct picture of energy differences between isomers for clusters containing fewer than ten atoms ${ }^{13}$. Because our LDA calculations are performed in real-space (see Methods), charged clusters are handled as easily as neutral ones. As an artificial uniform background density of the type used in plane-wave calculations is not used here, we do not expect charging of the clusters to result in any loss of LDA accuracy with respect to QMC. (3) It has been shown that introducing a gradient-corrected functional (providing for a more accurate estimate of the cohesive energy) does not change the relative energy ordering of either neutral or charged bare Si clusters, unless the LDA differences are on the milli-electron-volt level, that is, they are negligible ${ }^{19}$. Similar conclusions were obtained for nitrated Si clusters ${ }^{22}$. (4) We already mentioned that there is no universal agreement on the true ground-state structures in the literature. But even if the energy ordering presented here is wrong in some cases, the prior work cited above clearly agrees that, for some cluster sizes, isomers that are very close in energy do exist. 


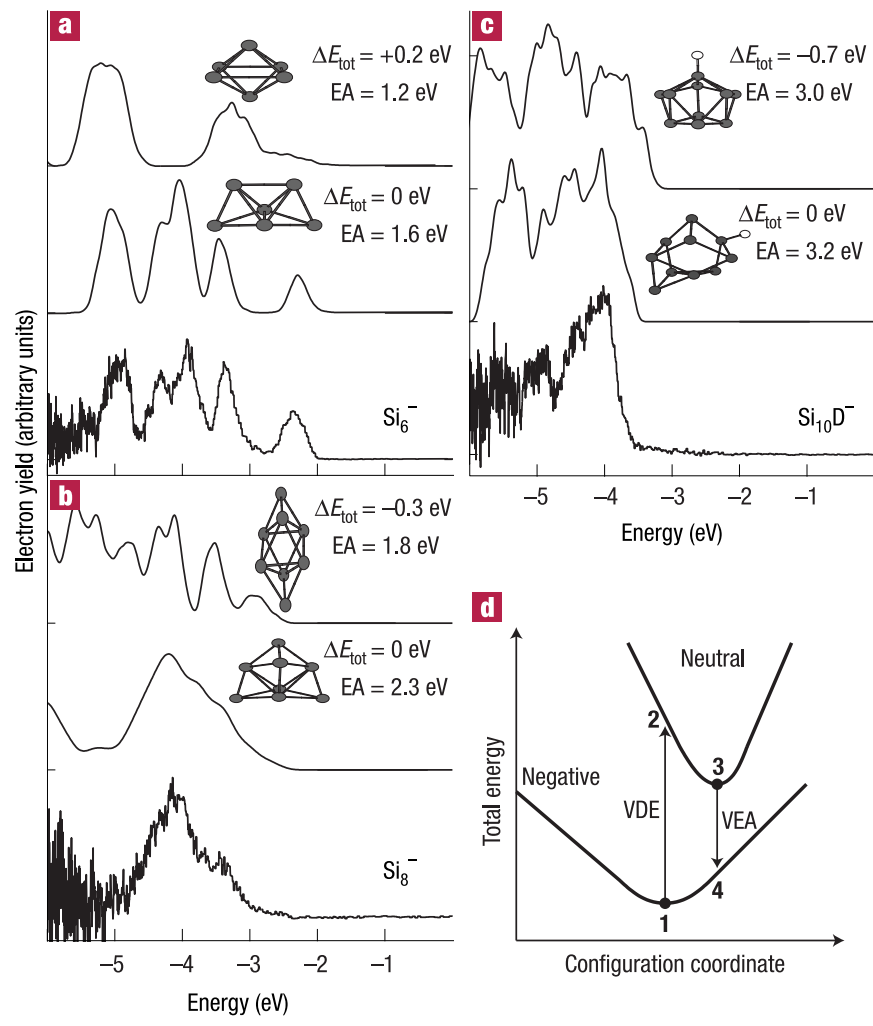

Figure 2 Photoelectron spectra of several clusters obeying the highest vertical electron affinity (VEA) rule. $\mathbf{a}, \mathrm{Si}_{6}{ }^{-}, \mathbf{b}, \mathrm{Si}_{8}{ }^{-}$and $\mathbf{c}, \mathrm{Si}_{10} \mathrm{D}^{-}$. Experimental PES data (bottom curves) are compared with theoretical PES data for the isomer yielding best agreement with experiment (centre curves). Also shown are theoretical PES data for the isomer of lowest total energy for which the computed curve disagrees with the experimental one (top curves). Structures, total energy differences ( $\Delta E_{\text {tot }}$, with respect to the isomer yielding best agreement with experiment) and electron affinities (EA) for those isomers are given as insets. d, Schematic configuration-coordinate diagram for the charged and neutral states that defines the vertical detachment energy (VDE) and the VEA (see text for discussion).

A failure of LDA would still not explain why experiment at room temperature clearly favours the observation of one isomer over the other, as in Fig. 2, whereas a mixture of PES 'signatures' of several isomers should have been expected. (5) It is equally unlikely that a 'wrong' energy ordering would result from an inexhaustive search of isomers. After all, the search did succeed in finding isomers that are in agreement with experiment for all cluster types studied.

If one rejects computational artifacts as the explanation of these results, then one must conclude that the observed isomers are often metastable, that is, their configuration corresponds to a local energy minimum that is not the ground state. Given that the room temperature ground-state structure should be very close to the zero temperature one, this indicates that thermodynamic equilibrium was not obtained experimentally in this case. This renders irrelevant any attempt to predict cluster structure theoretically, solely on the basis of minimum energy considerations.

Can one support the contention that we are dealing with a kinetically limited system? And if so, can one formulate an alternative, predictive criterion for metastable isomer observation? We believe that the key to such a criterion is the negative charge of the clusters. We assume that the observed clusters are initially formed as neutrals, with low-energy isomers formed at essentially equal probabilities due to the extremely high effective temperature during their formation. The clusters subsequently attract an electron from the plasma. If this is the case, the most probable process is for the electron to bind to the cluster with the highest VEA (the energy gain associated with a ' $3 \rightarrow 4$ ' transition in Fig. 2d). Electron attachment is thermodynamically allowed for all isomers with a positive VEA, but high VEA isomers are favoured by additional charge-transfer reactions between the anionic and neutral clusters ${ }^{23}$. In these reactions, electron 'hopping' from an isomer with a lower VEA to one with a higher VEA is energetically favourable, and can occur spontaneously. However, electron transfer in the other direction requires excess energy and would be curtailed by a fast removal of this energy via the He carrier gas. This is a well-known effect, for example, in oxide and fluoride impurities, where high VEA isomers appear in anion mass spectra with a higher relative abundance than in cation mass spectra. Thus, the net reaction rate will depend exponentially on the $\mathrm{VEA}^{24}$, making the highest VEA cluster the most favourable one.

On leaving the plasma region, the charged cluster cannot release the extra electron, and may have a dwell-time before measurement that would not allow it to surmount the energy barrier keeping the cluster in a metastable state. If the kinetic limitation is strong enough (that is, the cluster dwell-time is much shorter than its relaxation time), the cluster will instead simply relax to the metastable state of the highest VEA isomer (that is, complete the transition from 4 to 1 in Fig. 2d) and remain 'locked' in that state during measurement. The highest VEA would then replace the lowest total energy as the pertinent criterion for isomer selection. Naturally, the detailed kinetics may depend strongly on material and system specifics and need to be verified on a case-by-case basis.

To test our hypothesis for the present case, we have computed the VEA for all observed and unobserved isomers found. To do so, we relaxed the coordinates of the neutral cluster starting from charged geometry (that is, we followed the transition from 2 to 3 in Fig. 2d). We then subtracted the total energy of the charged cluster from that of the neutral isomer, using only the relaxed coordinates of the neutral isomer corresponding to the ' $3 \rightarrow 4$ ' transition in Fig. 2d. We found no cases where two distinct neutral isomers relaxed into the same charged one, or vice versa, so that each isomer studied was uniquely defined.

For 18 of the 20 cluster types studied, the observed isomer was indeed consistent with the highest VEA rule. The two exceptions are discussed and rationalized below. (In some cases, for example, $\mathrm{Si}_{10}{ }^{-}$, the PES spectra of different isomers were indistinguishable. Although such spectra do not lend active support to the highest VEA rule, they are consistent with it.) In particular, the electron affinities of the representative examples of Fig. 2 make the logic behind the experimental observation of some clusters but not others immediately obvious. This strongly supports our contention that the breakdown of the minimum energy rule here is not a failure of LDA but rather an indication of a kinetically limited process. It also indicates that the barrier height between metastable configurations is too high to overcome with the kinetic energy afforded by the energy gained from the electron capture.

The identification of the observed isomers as metastable ones also affects the interpretation of observed trends. For example, an inspection of the experimental spectra for $\mathrm{Si}_{8}{ }^{-}$(Fig. 2b) and $\mathrm{Si}_{8} \mathrm{D}_{2}{ }^{-}$ (Fig. 1d) may lead to the assumption that the introduction of deuterium has resulted in cluster passivation. This is because a HOMO-LUMO gap is observed for $\mathrm{Si}_{8} \mathrm{D}_{2}{ }^{-}$, but not for $\mathrm{Si}_{8}{ }^{-}$. However, inspection of Fig. $2 \mathrm{~b}$ reveals that the lowest-energy isomer of $\mathrm{Si}_{8}{ }^{-}$does feature an independent high-energy peak and therefore possesses a HOMO-LUMO gap ${ }^{21}$ even in the absence of deuterium. Therefore, passivation in the bulk electrical sense, that is, removal of states from the forbidden energy gap, does not seem to be applicable to such small clusters. 


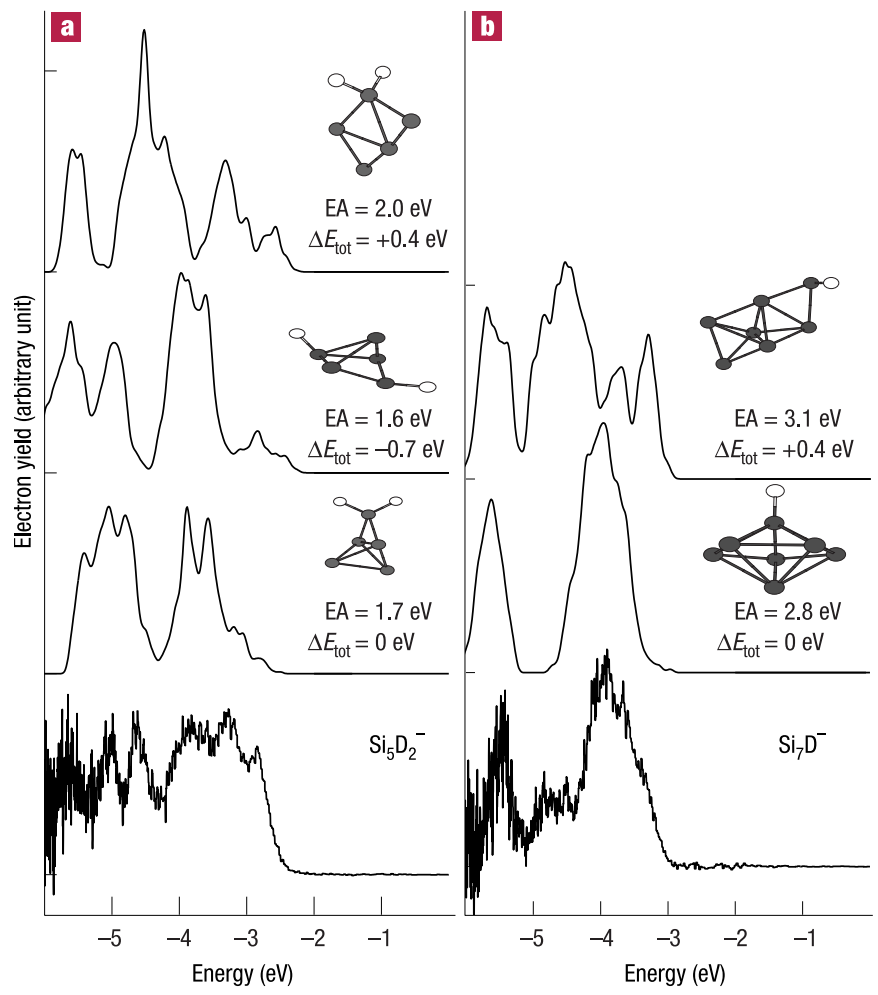

Figure 3 Photoelectron spectra of the two clusters disobeying the highest VEA rule. $\mathbf{a}, \mathrm{Si}_{5} \mathrm{D}_{2}{ }^{-}$. Theory: isomer of highest electron affinity (top curve), isomer of lowest total energy (second curve) and isomer yielding best agreement with experiment (third curve). Experiment-bottom curve. $\mathbf{b}, \mathrm{Si}_{7} \mathrm{D}^{-}$. Theory: isomer of highest electron affinity (top curve) and isomer yielding best agreement with experiment (centre curve). Experimentbottom curve. Structures, total energy differences ( $\Delta E_{\text {tot }}$, with respect to the isomer producing best agreement with experiment) and electron affinities (EA) for those isomers are given as insets.

The minimal total energy and the maximal VEA criteria represent two extreme limits for the dwell-time, both with respect to the cluster-relaxation time. The minimal total energy corresponds to a cluster dwell-time that is infinite. The maximal VEA corresponds to a dwell-time that is infinitesimal. In practice, the dwell-time is finite, and may allow for some isomer-transforming kinetics. We believe that such limited-range kinetics are the cause of the deviation from the electron affinity rule for two cluster types: $\mathrm{Si}_{5} \mathrm{D}_{2}{ }^{-}$and $\mathrm{Si}_{7} \mathrm{D}^{-}$.

For $\mathrm{Si}_{5} \mathrm{D}_{2}{ }^{-}$, the observable isomer was neither the highest VEA isomer nor the lowest energy one. This is readily seen in Fig. 3a, which compares PES spectra, structures, total energies and electron affinities for the highest affinity, lowest total energy and observed isomers. The highest affinity isomer yields a photoelectron spectrum with a large peak instead of a valley at about $-4.2 \mathrm{eV}$. The lowest energy isomer yields a photoelectron spectrum in which the energy positions of almost all peaks are in much worse agreement with experiment than those obtained from the observed isomer. Note that because cross-section effects are not explicitly included, the magnitude of the highest energy peak in the observed cluster may be underestimated by theory in this case, but good agreement for the peak position, and for both positions and intensities for other peaks, is obtained. The observed isomer is structurally close to the highest affinity one. The two deuterium atoms are bound to the same silicon atom, and the two isomers can be obtained from one another by shifting one silicon atom. Isomers not possessing this double-deuterium structure, such as the lowest energy isomer, did not agree with experiment as well. A plausible explanation is that the cluster dwell-time did allow for a limited relaxation to a closely related structure, but not to the ground state.

$\mathrm{Si}_{7} \mathrm{D}^{-}$presented the only case where the best agreement with experiment was obtained from the lowest total energy isomer and not from the highest VEA isomer, as shown in Fig. 3b. The bi-capped pentagonal pyramid ground-state structure of $\mathrm{Si}_{7}{ }^{-}$is well known to be particularly stable ${ }^{16}$. This structure is retained in $\mathrm{Si}_{7} \mathrm{D}^{-}$, with an extra deuterium cap at the top of the pyramid (Fig. 3b). It is conceivable that $\mathrm{Si}_{7} \mathrm{D}^{-}$is also particularly stable, and that this stability is associated with many reaction pathways leading to the formation of the ground-state structure. It is then likely that the cluster dwell-time, which was too short for the complete relaxation of other clusters, was sufficient for obtaining the complete relaxation of $\mathrm{Si}_{7} \mathrm{D}^{-}$. It is still possible that traces of the highest electron-affinity isomer are manifested in the experimental spectrum of $\mathrm{Si}_{7} \mathrm{D}^{-}$, as the small shoulder between $-4.8 \mathrm{eV}$ and $-4.5 \mathrm{eV}$. This is because the highest-affinity isomer has particularly strong peaks in that energy region, whereas the minimum energy one is devoid of a meaningful signal in this region.

In conclusion, our findings point to a need for a stringent examination of the traditional assumption of thermodynamically limited cluster formation, and for appropriate modifications in the interpretation when the thermodynamic limit is not realized experimentally.

\section{Methods}

Negatively charged $\mathrm{Si}_{m} \mathrm{D}_{n}$ clusters ( $4 \leq m \leq 10,0 \leq n \leq 2$, D denoting deuterium) were produced using a pulsed arc cluster ion source ${ }^{25}$. Bulk silicon was vapourized in a pulsed arc, with the formed hot plasma flushed by $\mathrm{He}$ carrier gas into an extender $20 \mathrm{~cm}$ long and $4 \mathrm{~mm}$ in diameter. For the generation of deuterated clusters, molecular deuterium was introduced into the extender. Owing to partial molecular dissociation in the arc, a considerable amount of atomic deuterium was present in the extender ${ }^{26}$. Clusters were cooled down to approximately room temperature and flushed into vacuum at the extender exit. No further annealing was applied ${ }^{27}$.

Negatively charged clusters were mass selected in a time-of-flight mass spectrometer. Photoelectron spectra were recorded by irradiating the mass-selected clusters with an ultraviolet laser pulse (ArF excimer laser, $6.4 \mathrm{eV}$ photon energy, energy flux of $50 \mathrm{~mJ} \mathrm{~cm}^{-2}$ ) and measuring the kinetic energy of the photoelectrons using a 'magnetic bottle'-type time-of-flight electron spectrometer, with a relative energy resolution of $\Delta E / E \approx 1 \%$. For the electron kinetic energies of $1-2 \mathrm{eV}$ found experimentally, this corresponded to an absolute energy resolution of $10-20 \mathrm{meV}^{28}$. The absolute energy accuracy was $\sim 0.2 \mathrm{eV}$, owing to space charge effects and to changes in the work function of the surfaces within the electron spectrometer (caused by the intense cluster ion and laser beams).

Deuturium, rather than hydrogen, was used in this study to alleviate the problem of contamination of the mass selection by heavy silicon isotopes. However, this does not remove the problem completely (for example, $\mathrm{Si}^{30}$ has the same mass as $\mathrm{Si}^{28} \mathrm{D}$ ). We therefore measured the spectrum of the bare $\mathrm{Si}_{m}{ }^{-}$clusters first, and then identified bare-cluster-related features in the $\mathrm{Si}_{m} \mathrm{D}^{-}$spectra. A weighted version of the spectrum of the bare cluster was then substracted so that all features assigned to it were removed from the $\mathrm{Si}_{m} \mathrm{D}^{-}$spectrum. A similar procedure was then used to remove $\mathrm{Si}_{m}{ }^{-}$and $\mathrm{Si}_{m} \mathrm{D}^{-}$features from the $\mathrm{Si}_{m} \mathrm{D}_{2}{ }^{-}$ spectra. Trivially, this requires that the spectra of $\mathrm{Si}_{m} \mathrm{D}_{n}{ }^{-}$clusters with equal $m$ but different $n$ be significantly different. This was indeed the case for all the data presented here.

All computations presented were based on solving the Kohn-Sham DFT equations within the LDA, using the higher-order finite-difference pseudopotential $\operatorname{method~}^{29}$. For obtaining low-energy metastable isomers of the clusters, we used the simulated annealing approach, implemented within the framework of Langevin molecular dynamics followed by a strict minimization of energy ${ }^{4}$. A detailed account of the computational approach has been provided elsewhere ${ }^{12}$.

Theoretical photoelectron spectra were computed within the constant matrix approximation, that is, cross-sectional effects on the computed density-of-states (DOS) curves were neglected. Because the experimental data were taken from the highest-energy valence states, which derive predominantly from $\mathrm{Si}$ $3 p$ orbitals ${ }^{14}$, and because cross-section effects are primarily orbital dependent ${ }^{30}$, this is a reasonable approximation.

To facilitate comparison with experiment, the computed PES curves were broadened without an explicit use of a broadening parameter by averaging the DOS over several picoseconds of isothermal molecular dynamics ${ }^{14}$. Use of a temperature of $750 \mathrm{~K}$ in the Langevin equation of motion was empirically found to yield a broadening similar to the experimental one. This temperature is higher than the actual experimental temperature (which was around room temperature), because it compensates for FranckCondon broadening that is not explicitly included in the computation.

For an anionic cluster, the energy of the highest-lying spectral peak in the photoelectron spectrum corresponds to its vertical detachment energy (VDE), that is, the energy needed to remove an electron from the highest occupied orbital without relaxing the rest of the system ${ }^{15}$. This is the energy barrier to the ' $1 \rightarrow 2$ ' transition in Fig. $2 \mathrm{~d}$. The VDE is readily obtained theoretically by subtracting the total energy of the relaxed negatively charged isomer from that of its neutral counterpart of the same geometry. All theoretical PES curves were aligned according to the theoretical VDE, as in ref. 15. For obtaining the best agreement with experiment, we allowed only for an additional small rigid shift of up to $0.2 \mathrm{eV}$,

compensating for the experimental uncertainty discussed above. 


\section{References}

1. Jellinek, J. (ed.) Theory of Atomic and Molecular Clusters : with a Glimpse at Experiments (Springer, Berlin, 1999).

2. Kawazoe, Y., Kondow, T. \& Ohno, K. Clusters and Nanomaterials: Theory and Experiment (Springer, Berlin, 2001).

3. Car, R. \& Parrinello, M. Unified approach for molecular dynamics and density-functional theory. Phys, Rev. Lett. 55, 2471-2474 (1985).

4. Binggeli, N., Martins, J. L. \& Chelikowsky, J. R. Simulation of Si clusters via Langevin molecular dynamics with quantum forces. Phys. Rev. Lett. 68, 2956-2959 (1992).

5. Barnett, R. N. \& Landman, U. Born-Oppenheimer molecular-dynamics simulations of finite systems: Structure and dynamics of $\left(\mathrm{H}_{2} \mathrm{O}\right)_{2}$. Phys. Rev. B 48, 2081-2097 (1993).

6. Marzari, N., Vanderbilt, D. \& Payne, M. C. Ensemble density-functional theory for ab initio molecula dynamics of metals and finite-temperature insulators. Phys. Rev. Lett. 79, 1337-1340 (1997).

7. Geissler, P. L., Dellago, C., Chandler, D., Hutter, J. \& Parrinello, M. Autoionization in liquid water. Science 291, 2121-2124 (2001).

8. Passerone, D. \& Parrinello, M. Action-derived molecular dynamics in the study of rare events. Phys. Rev. Lett. 87, 108302 (2001)

9. Ho, K.-M. et al. Structures of medium-sized silicon clusters. Nature 392, 582-585 (1998)

10. Wales, D. J. \& Scheraga, H. A. Global optimization of clusters, crystals, and biomolecules. Science $\mathbf{2 8 5}$, 1368-1372 (1999).

11. Wang, B., Yin, S., Wang, G., Buldum, A. \& Zhao, J. Novel structures and properties of gold nanowires Phys. Rev. Lett. 86, 2046-2049 (2001)

12. Kronik, L., Vasiliev, I., Jain, M. \& Chelikowsky, J. R. Ab initio structures and polarizabilities of sodium clusters. J. Chem. Phys. 115, 4322-4322 (2001).

13. Grossman, J. C. \& Mitas, L. Quantum Monte Carlo determination of electronic and structural properties of $\mathrm{Si}_{{ }_{1}}$ clusters $(n \leq 20)$. Phys. Rev. Lett. 74, 1323-1326 (1995).

14. Binggeli, N. \& Chelikowsky, J. R. Photoemission spectra and structures of Si clusters at finite temperature. Phys. Rev. Lett. 75, 493-496 (1995).

15. Müller, J. et al. Spectroscopic evidence for the tricapped trigonal prism structure of semiconductor clusters. Phys. Rev. Lett. 85, 1666-1669 (2000).

16. Raghavachari, K. \& Rohlfing, C. M. Electronic structure of the negative ions $\mathrm{Si}_{2}{ }^{-}-\mathrm{Si}_{10}{ }^{-}$: electron affinities of small silicon clusters. J. Chem. Phys. 94, 3670-3678 (1991).

17. Wei, S., Barnett, R. N. \& Landman, U. Energetics and structures of neutral and charged $\operatorname{Si}_{n}(n<\sim 10)$ and sodium-doped $\mathrm{Si}_{1}$ Na clusters. Phys. Rev. B 55, 7935-7944 (1997).
18. Xu, C., Taylor, T. R., Burton, G. R. \& Neumark, D. M. Vibrationally resolved photoelectron spectroscopy of silicon cluster anions $\mathrm{Si}_{n}{ }^{-}(n=3-7)$. J. Chem. Phys. 108, 1395-1406 (1998).

19. Shvartsburg, A. A., Liu, B., Jarrold, M. F. \& Ho, K.-M. Modeling ionic mobilities by scattering on electronic density isosurfaces: application to silicon cluster anions. J. Chem. Phys. 112, 4517-4526 (2000).

20. Xu, C., Taylor, T. R., Burton, G. R. \& Neumark, D. M. Photoelectron spectroscopy of $\mathrm{Si}_{n} \mathrm{H}(n=2-4)$ anions. J. Chem. Phys. 108, 7645-7652 (1998).

21. Chesnovsky, O. et al. Ultraviolet photoelectron spectroscopy of semiconductor clusters: silicon and germanium. Chem. Phys. Lett. 138, 119-124 (1987).

22. Jurnickel, G., Fraunheim, T. \& Jackson, K. A. Structure and energetics of $\mathrm{Si}_{n} \mathrm{~N}_{m}$ clusters: growth pathways in a heterogenous cluster system. J. Chem. Phys. 112, 1295-1305 (2000)

23. Boltalina, O. V., Dashkova, E. V. \& Sidorov, L. N. Gibbs energies of gas-phase electron transfer reactions involving the larger fullerene anions. Chem. Phys. Lett. 256, 253-260 (1996).

24. Hill, T. L. Introduction to Statistical Thermodynamics Ch. 11 (Dover, New York, 1986).

25. Cha, C. Y., Ganteför, G. \& Eberhardt, W. New experimental setup for photoelectron spectroscopy on cluster anions. Rev. Sci. Instrum. 63, 5661-5666 (1992).

26. Burkart, S. et al. Experimental verification of the high stability of $\mathrm{Al}_{13} \mathrm{H}$ : A building block of a new type of cluster material? Chem. Phys. Lett. 301, 546-550 (1999).

27. Handschuh, H., Ganteför, G., Kessler, B., Bechthold, P. S. \& Eberhardt, W. Stable configurations of carbon clusters: chains, rings, and fullerenes. Phys. Rev. Lett. 74, 1095-1098 (1995).

28. Handschuh, H., Ganteför, G. \& Eberhardt, W. Vibrational spectroscopy of clusters using a "magneti bottle" electron spectrometer. Rev. Sci. Instrum. 66, 3838-3843 (1995).

29. Chelikowsky, J. R., Troullier, N. \& Saad, Y. Finite-difference-pseudopotential method: Electronic structure calculations without a basis. Phys. Rev. Lett. 72, 1240-1243 (1994)

30. Woicik, J. W. et al. Hybridization and bond-orbital components in site-specific photoelectron spectra of rutile $\mathrm{TiO}_{2}$. Phys. Rev. Lett. 89, 74401 (2002).

\section{Acknowledgements}

We acknowledge the support provided by the National Science Foundation, the US Department of Energy, the Minnesota Supercomputer Institute, the Deutsche Forschungsgemeinschaft and the Sonderforschungsbereich 513

Correspondence and requests for materials should be addressed to J.R.C.

\section{Competing financial interests}

The authors declare that they have no competing financial interests. 